\title{
EXECUTIVE CONTROL INFLUENCED BY THE TIME COURSE OF
} ALERTING EFFECT

\author{
LU Xiuling $^{1,2}$, SUN Xianghong ${ }^{1}$ ZHANG Kan ${ }^{1}$ \\ ${ }^{1}$ State Key Laboratory of Brain and Cognitive Science, Institute of Psychology, \\ Chinese Academy of Sciences, 4A Datun Road, Chaogyang District, Beijing, China, \\ 100101; PH (+86) 6483-7096; FAX (+86) 6483-7182; email: zhangk@psych.ac.cn \\ ${ }^{2}$ Graduated School, Chinese Academy of Sciences, Beijing 100039, China.
}

\begin{abstract}
Inattention has been shown to be the greatest factor in the cause of driving collision and the warning signal can abstract the drivers' attention to the driving performance so that to reduce the driving collision.. Driving is a complex task that taps mechanisms of cognition and attention. The understanding of the mechanisms of cognition and attention is useful to the understanding of driving. As Posner et al proposed the attention function is carried out by three different attention networks: alerting, orienting and executive control. The question is that whether the arousal level can affect executive control. The answer to this question can explain the effect of warning in driving and be useful to the design of warning signal. In the present experiment we used a modified version of the attention network test (ANT) to assess the interaction of alerting and executive control. We found that, when the alerting function is highly activated, the executive control prevents the system from engaging in higher level processing to enhance the responses to the present stimulus. And their inhibitory relationship is affected by the alert-cue interval. This result suggests us that we should pay attention to the warning signal time in driving.
\end{abstract}

Key words: Alerting, Attention network, Executive control, Orienting.

\section{Introduction}

Inattention has been shown to be the greatest factor in the cause of driving collision (Daniel J. Cox, et al, 2006). No clear medical definition of inattention. From the dictionary, "inattention” can be interpreted as "unilateral neglect." In other words, the individual attends to another activity or selectively ignores a particular sensory input. Essentially, when one concentrates on one task, he or she might ignore other tasks. For those tasks, the subject is inattentive (Eberhart, R, et al, 2000).The warning signal can draw the drivers' attention to the driving performance so that to reduce the driving collision. Driving is a complex task that taps mechanisms of cognition and attention. The understanding of the mechanisms of cognition and attention is useful to the understanding of driving.

Several authors have argued that the attention function is carried out by three different attention networks. These networks were named the alerting network, orienting network, executive control network (Posner MI, Petersen SE, 1990). The alerting network prepares the system for fast reactions by means of a change in the 
internal state. The alerting signal tells the subject that a target will occur but usually provides little or no information about where it will be (Fernandez-Duque D, Posner M I, 1997). The orienting network selectively allocates attention to a potentially relevant area of the visual field and/or object, and it enhances its perceptual processing. The executive control network is thought to be active when the cognitive system faces situations that involve planning, making a decision, detecting an error, giving a novel response or overcoming habitual actions (Norman DA, Shallice T, 1986).

The three attentional networks have been studied under behavioral, neuropsychological and functional perspectives (Posner MI, Petersen SE, 1990; Fan J,et al,2002;Callejas A,et al, 2004,2005;Fuentes, LJ., Campoy, G, 2007). Although the interactions between the alerting and orienting, orienting and executive control are well established, the interaction between alerting and executive control is rather controversial. The question is that whether the arousal level can affect executive control. The answer to this question can explain the effect of warning in driving and be useful to the design of warning signal.

Posner proposed an inhibitory relationship between the alerting and executive function networks which means when the alerting function is highly activated, the executive control prevent the system from engaging in higher level processing to enhance the responses to the present stimulus. He termed this state as "clearing of consciousness” (Posner MI, 1994).

Some researchers used original the attention network test (ANT) not to find any interaction between alerting and executive control (Fan J,et al,2002; Roberts, K. L,et al, 2006), however. One reason why these studies did not find the interactions was that the ANT uses asterisks for both alerting and orienting (Callejas A, et al, 2004, 2005) . So Callejas et al modified the ANT. In the modified ANT, an alerting sound was introduced and two of the four levels of the original cueing variable were eliminated in order to be able to separately measure alerting and orienting. However, the authors also didn't find the interaction between alerting and executive control (Callejas A,et al, 2004,2005;Fuentes, LJ., Campoy, G, 2007).

The absence of interaction under these conditions let us to think that the modality of alert and the temporal course of alerting effect might be key factors influencing the interaction between alerting and executive control. We proposed that, on one hand, the intensity of alerting effect was different with the temporal course and with the different modalities of alert; on the other hand, the congruent effect would be different in different intensity of alerting effect. For this reason, we conducted the experiment in which there was auditory alert compared with visual alert, and the alert-cue SOA included four values of $50 \mathrm{~ms}, 100 \mathrm{~ms}, 300 \mathrm{~ms}$ and $500 \mathrm{~ms}$. 
Eighteen undergraduates participated in the experiment. None of the participants had prior experience with any version of the ANT, and all had normal or corrected-to-normal vision and hearing.

\subsection{Stimuli and apparatus}

Stimuli were displayed on a 17- CTR monitor, which was set to a screen resolution of $640 \times 480$ pixels. Responses were collected through the computer keyboard, and headphones were used to present the alerting tones.

The stimulus used for the orienting signal was an asterisk presented at the same location as the target $\left(1.06^{\circ}\right.$ of visual angle above or below fixation point). For the auditory alerting signal, a $300 \mathrm{~Hz}$ and $50 \mathrm{~ms}$ tone was used. For the visual alerting signal, a $0.01^{\circ}$ and $50 \mathrm{~ms}$ red point was used. The target display was made up of a target arrow that could point either to the left or to the right, and four flankers arrows pointing either left or right. The length of the arrows was $0.55^{\circ}$ and they were $0.06^{\circ}$ away from each other.

\subsection{Procedure}

Participants were seated approximately $53 \mathrm{~cm}$ from the computer screen, and were instructed to respond to the direction of the target stimulus by pressing one of two possible keys in the keyboard. Feedback regarding accuracy was given during the practice block, but not during the experimental blocks. Participants could rest between blocks. The mapping of hand-response was always compatible (a rightward arrow was to be responded to with the right hand and a leftward arrow with the left hand).

The experiment consisted of four blocks of 144 trials. In each block, all possible combinations of the alerting condition (auditory alert, visual alert, no alert), alert-cue SOA (50, 100, 300, $500 \mathrm{~ms}$ ), cuing condition (valid cue, invalid cue, no cue), and flanker type (congruent, incongruent) were presented once. Thus, there were eight trials per experimental condition in the whole experiment. Target location (above, below) and target direction (right, left) were balanced but not considered as experimental factors. The visual alert, auditory alert and no alert were equal in the trials.

The basic configuration described above was presented for a variable duration between 1,200 and 2,600 ms, being the precise duration determined randomly, with the constraint that the entire range was homogeneously represented within each block of trials. The visual and auditory alert were presented for $50 \mathrm{~ms}$. In the no alert condition, there were empty audio for $50 \mathrm{~ms}$. After alert-cue SOA (50, 100, 300, 500 $\mathrm{ms}$ ), a cue was presented on $2 / 3$ of the trials for $50 \mathrm{~ms}$. $1 / 3$ of the times, the cue was presented on the location of the target (valid cued trials) and 1/3of the times on the location opposite to that of the target (invalid cued trials).The orienting visual cue appeared for $50 \mathrm{~ms}$ in the central box of either the upper or the lower box row. Finally, with cue-target SOA of $350 \mathrm{~ms}$, the targets were presented. They were presented until the participant indicated whether the target arrow pointed to the right 
or to the left by pressing key $\mathrm{V}$ or $\mathrm{M}$, respectively. The target arrows pointed to the right in half of the trials and to the left in the other half. Flanker arrows could point to the same direction of the target (the congruent condition) in half of the trials, or to the opposite direction of the target (the incongruent condition) in the other half. Participants were instructed to respond as fast and accurate as possible.

\section{Result}

Accuracy was very high in this experiment. Therefore, data analyses were carried out only on RTs. In each condition, RTs above or below 3 SD from the mean were not included in the statistical analyses (1.40\% of the data).

Correct RTs were submitted to a repeated measures analysis of variance (ANOVA), with alerting (auditory alert, visual alert, no alert), alert-cue SOA (50, 100, 300, 500ms), cuing (valid cue, invalid cue, no cue), and congruency (congruent, incongruent) as the within-subjects factors. The results showed significant main effects for all factors. $\mathrm{F}(2,16)=17.14, \mathrm{p}<.001$ for alerting, responses were faster for auditory and visual alerting trials than for no alert trials , and there was no significant different between auditory alert and visual alert. $\mathrm{F}(3,15)=5.179, \mathrm{p}<.05$ for SOA, responses were lower for $500 \mathrm{~ms}$ trails than for $100 \mathrm{~ms}$ and $300 \mathrm{~ms}$ trials, and there was no significant different among other trails. $\mathrm{F}(2,34)=71.95, \mathrm{p}<.001$ for cuing, responses were faster for valid cue trails than for invalid cue and no cue trials, and there was no significant different effect between invalid cue and no cue trails. F (1, $17)=478.40, p<.001$ for congruency, responses were faster for congruent trails than incongruent trails.

Interactions between the factors were found here. The interaction between alerting and SOA was significant, $[\mathrm{F}(6,12)=8.47, \mathrm{P}<0.001]$. The reaction time of no alert was almost equal in the four alert-cue SOA level, but to auditory alerting trails and visual alerting trail, responses were faster in 100 and $300 \mathrm{~ms}$ than in $50 \mathrm{~ms}$ and 500ms. The auditory alerting effect (difference between auditory alert and no alert) and visual alerting effect (difference between visual alert and no alert) present a " $\cap$ ” shape with the four alert-cue interval.

The interaction between alerting and cuing was significant, $[\mathrm{F}(4,14)=6.96, \mathrm{P}<$ 0.001]. In the invalid cued trails and valid cued trails, responses of the different alert trails were almost equal. But in the no cue trails, the reaction time of auditory alerting and visual alerting trails was faster than in the no alerting trails.

The interaction between cuing and congruency was significant, $F(2,14)=4.214$, $\mathrm{P}<0.05$. We found a larger congruency effect (difference between congruent and incongruent trials) in the invalid cued trails than in the valid cued trails.

We found a marginal significant interaction between SOA and congruency, F (3, $15)=2.69, \mathrm{P}=0.08$. The congruent effect present a "V" shape with the four SOA level.

No other effects reached statistical significance.

\section{Discussion of Results}


The aim of the experiment reported in this paper was to clarify the interaction between alerting and executive control in the ANT task. First, we used auditory alerting signal and visual alerting signal to verify if the modality of alerting signal produced the previous results. Second, we wanted to know whether there were interaction between alerting and executive in the ANT task. We manipulated alert-cue interval at four SOA values $(50,100,300,500 \mathrm{~ms})$ to trace the alerting network influence over the executive control network.

Here we found there was not significant difference between auditory alert trails and visual alert trails. And we also found that the alerting effect presents " $\cap$ ” shape with the four alert-cue intervals. Meanwhile, the response of congruent trials was equal in the four alert-cue intervals, but the response of incongruent trails presents a " $V$ " shape. That means the congruent effect was least when the alerting effect was high.

As proposed by Posner, the alerting network enhance fast responses to sensory input in order to detect an infrequent target and prevent the system from focusing on feelings or thoughts or on further processing of the stimulus(Posner MI, 1994). This was claimed as "inhibitory" effect: the alerting network influences the executive control network by inhibiting its function, so that the organism can focus on giving a fast response rather than concentrating on control. This inhibition was observed in our results as a fast response for incongruent trials when the alerting effect was high. Thus, the inhibition of the executive function network in these trials was observed.

Interesting, in this experiment, we didn't found the direct interaction between alerting type and congruency but we found the interaction between SOA and congruency. This was of great importance, considering the unclear previous findings in the literature (Fan J,et al,2002;Callejas A,et al, 2004,2005;Fuentes, LJ., Campoy, G, 2007; Roberts, K. L,et al, 2006). In order to fully understand the results obtained in this experiment, it was important to refer to our previous findings in which the interaction was not found in tasks exactly the same as this but with an auditory alert and a alert-cue SOA of $450 \mathrm{~ms}$.

\section{Conclusions}

In precious ANT tasks, the authors didn't find the interactions between alerting and congruency. Though, we also didn't found the interaction between the two, we found the interaction between SOA and congruency. Considering the SOA influenced the alerting effect, we conclude that the alerting intensity affects the executive control. This result answers the unclear previous findings in the literature (Fan J,et al,2002;Callejas A,et al, 2004,2005;Fuentes, LJ., Campoy, G, 2007; Roberts, K. L,et al, 2006). However, we got this conclusion from the fact that the alert-cue SOA influenced the alerting effect.

This result supports the inhibitory relationship between the alerting and executive function networks, which means, when the alerting function is highly activated, the executive control prevent the system from engaging in higher level 
processing to enhance the responses to the present stimulus. And their inhibitory relationship is affected by the alert-cue interval.

Future research will manipulate the intensity of the alert signal, such as the diameter of visual alert; the fluency, the pitch of auditory alert. Through this manipulation, we want to clarify how the intensity of alerting effect influences over the executive control.

Meanwhile, this conclusion suggests us that we should pay attention to the warning signal time in driving. In the future, we will observe how the alerting effect affects driving performance using the ANT and how the SOA of warning signal affects driving performance.

\section{References}

Callejas A, Lupiáñez J, Tudela P. (2004). The three attentional networks: on its independence and interactions. Brain Cognition, vol. 54, pp 225-227.

Callejas A, Lupiàñez J, Funes MJ, Tudela P. (2005). Modulations among the alerting, orienting and executive control networks. Exp Brain Res, vol.167, pp27-37.

Cox, DJ, Punja, M, Powers, K, Merkel, RL, Burket, R; Moore, M. (2006) Manual transmission enhances attention and driving performance of ADHD adolescent males: Pilot study. Journal of Attention Disorders,10(2), 212-216.

Eberhart, R., Hu, X., \& Foresman, B. (2000). Dangers of sleepiness and inattention while driving. Journal of the American Osteopathic Association, 100(8), S9-S14.

Fan J, McCandliss BD, Sommer T, Raz A, Posner MI. (2002). Testing the efficiency and independence of the attentional networks. J Cogn Neurosci, vol.4, pp340-347.

Fernandez-Duque D and Posner M I. (1997). Relating the mechanisms of orienting and alerting. Neuropsychologia, vol.35, pp477-486.

Fuentes, L. J., Campoy, G. (2007). The time course of alerting effect over orienting in the attention network test. Exp Brain Res, vol. 185, pp667-672.

Posner MI, Petersen SE. (1990). The attention system of the human brain. Annu Rev Neurosci, vol. 13, pp25-42.

Posner MI. (1994). Attention: the mechanisms of consciousness. Proc Natl Acad Sci USA, vol. 97, pp7398-7403.

Norman DA, Shallice T. (1986). Attention to action: willed and automatic control of behavior. In: Davidson RJ, Schwartz GE, Shapiro D (eds) Consciousness and self-regulation. Plenum, New York, pp1-18.

Roberts, K. L., Summerfield, A. Q., \& Hall, D. A. (2006). Presentation modality influences behavioural measures of alerting, orienting, and executive control. Journal of the International Neuropsychological Society, vol. 12, pp485-492. 